\title{
Cross-Layer Design Approach for Multicast Scheduling over Satellite Networks
}

\author{
A.Sali, A.Widiawan, S. Thilakawardana, R.Tafazolli, B.G.Evans \\ Mobile Communications Research Group, Centre for Communications Systems Research, \\ University of Surrey, \\ GU2 7XH Guildford, Surrey, \\ UK
}

\begin{abstract}
In this paper, we propose a cross-layer design approach using perfect prediction-based wireless channel conditions to improve the performance of a multicast packet scheduler over satellite network environments in the downlink transmission. The satellite channels are modeled in single and multi-environments with different values of Rician $K$ factors and its corresponding elevation angle, mean and standard deviation values. From simulation results, the channel state information (CSI) of each user in the multicast group is considered and becomes the condition for the transmission of the multicast packet. We assume the users suffer slow-varying channels such that the CSI update is within the time interval for slot allocation. The result indicates that a positive performance improvement is gained by adopting a cross-layer-design approach in a fading environment. By obtaining the CSI before transmitting multicast flows, the approach reduces unnecessary transmission of background traffic and hence reduces unnecessary resource allocation and retransmission requests.

Index Terms - cross-layer design, channel-state-information,
\end{abstract} multicast scheduler, geo-stationary satellite networks

\section{INTRODUCTION}

$\mathrm{R}$ esearch on cross-layer design has recently attracted significant interest. It is concerned with adapting or sharing of information among various layers as specified in the open system interconnection (OSI) protocol layers. There are a number of papers in the literature addressing specific cross-layer issues. In [1], channel variation and traffic burstiness is exploited to improve the performance of resource allocation. The merging of the two parameters has shown tremendous increase in network performance. Crosslayer design mechanism can be easy and simple to implement, as discussed in [8]. The authors merge the scheduling information in medium access control (MAC) layer with rate selection in physical/link (PHY/LINK) layer. The mechanism is implemented by using one single bit ACK/NACK signal indicating the correct reception/failure of the packet in downlink transmission. Another instance of a cross-layer scheduler that enjoys low-complexity implementation can be found in [5]. The paper develops cross-layer design for multiuser scheduling at the data link layer with each user employing adaptive modulation and coding (AMC) at the PHY layer. The scheduler classifies users in two categories: QoS guaranteed and best-effort. The scheduler has the capability to enable prescribed QoS guarantees and efficient bandwidth utilization simultaneously.

Satellite networks offer the advantage of wide geographical coverage. To model the satellite propagation channel, [2] uses two-level Markov state method. The propagation fading is treated as slow fading (shadowing) and fast fading (multipath). Two Markov state transition matrices are used to simulate the variation in the strength of the received signal caused by shadowing or multipath fading. In [7], channel characterization for mobile satellite communications is presented. The channel model is suitable for quasi-stationary channels, which is implemented in this paper. Quasi-stationary channels are defined as channels characterized by slowly varying environmental conditions.

In [3], it is indicated that the lack of channel state information in the satellite interface differentiates the packet scheduler in the satellite interface to its terrestrial counterpart. The authors adapt weighted fair queuing (WFQ) and multilevel priority queuing (MLPQ) mechanisms for the timescheduling function. The work has achieved significant results in terms of resource utilization achieved by the scheduler and performance obtained by the flows at the packet level.

In multicast transmission, the delivery mechanism sends a packet to all multicast group users using one resource unit instead of $N$ resource units to $N$ users in unicast transmission. However, in multicast transmission even if one of the users in a multicast group requests a retransmission due to bad channel condition, the retransmission process has to be executed to all users in the group. This scenario will exhaust the network resource. To reduce retransmission requests, we propose to adapt channel state information (CSI) as a conditional decision to resume packet transmission.

In this paper, we propose an adaptation of physical layer channel state information onto the multicast packet scheduler at the MAC layer to improve network performance in a geostationary satellite system. We consider downlink transmission from the satellite to the multicast group users.

This paper is organized as follows. In Section II we present the system architecture of the proposed multicast scheduler with cross-layer design approach. The initial simulation results are discussed in Section III. Finally we summarize our current conclusion and propose future research direction in Section IV. 


\section{SYSTEM ARCHITECTURE}

\section{A. Multicast packet scheduling with channel state information}

The adaptation of the proposed cross-layer design approach is based on sharing the channel status information from physical layer with the multicast packet scheduler at the MAC layer. Having access to this information the scheduler can make the decision as to whether the channel is relatively 'good' or 'bad' prior to resuming transmission. If a 'bad' channel is predicted, the associated packet will be paused until it sees a 'good' channel status.

The system that we address in this paper handles fixed packet size, $l_{p}$, which is set to 125 bytes in a TDMA environment and each TDMA slot is $20 \mathrm{~ms}$. We characterize two distinct classes of multicast service: streaming and file delivery.

Streaming traffic, being non-delay tolerant, will always be allocated slots and transmitted to the users regardless of the channel information. Thus only file delivery services, which are not delay-restricted, will be considered for channel-statedependent scheduling. We summarize our scheduling policy as follows:

\section{Scheduling and Admission Policy}

1) For each streaming traffic packet, time slots are allocated at all time.

2) For the remaining slots, if file delivery traffic packets arrive, channel state information (CSI) for the intended multicast group is calculated.

3) If CSI is above a certain threshold, the slot is allocated to that packet. If not, then the slot is allocated to another service such as unicast.

The scheduling policy is illustrated in Figure 1. Referring to the figure, the channel state information for each receiver is obtained from the physical layer every $20 \mathrm{~ms}$ for slot allocation. The algorithm to obtain the channel information is as follows:

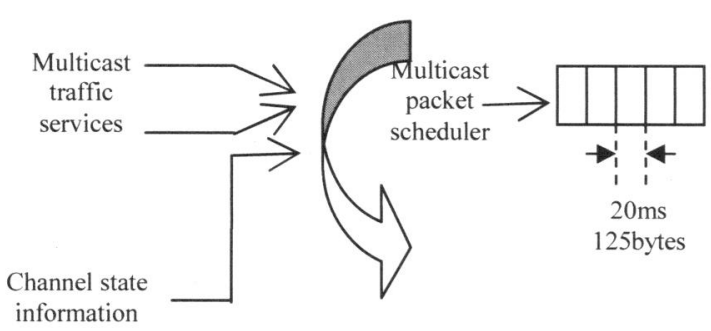

Fig. 1 Multicast scheduler with channel-state-dependence

1) Channel level, $s_{i}(t)$, for each multicast group user $\mathrm{i}$ is calculated according to lognormal fading as defined in (1).

2) This channel information corresponds to packet error rate $\left(\mathrm{PER}_{\mathrm{i}}\right)$ from lookup table obtained from a PER performance reference curves [6].

3) Threshold ( PER $_{\text {thresh }}$ ) for each receiver is established on the strength of the fading at that particular instant, according to packet error rate (PER) stated in Table I. In the simulation, file delivery service with PER $10^{-2}$ is assumed.

4) Depending on comparison of $P E R_{i}$ and $P E R_{\text {threshold, a }}$ decision is made as to whether the channel is in 'good' or 'bad' condition.

5) The percentage of users in a multicast group, $\varsigma_{i}$, achieving PER higher than the threshold, $\varsigma_{T}$, is calculated.

6) If the percentage, $\varsigma_{i}$, is greater than the specified threshold, $\varsigma_{T}$, then the packet is selected for transmission

7) If not, then the packet is delayed until percentage of users with 'good' channel condition is achieved. Whilst the packets are delayed, the resource can be utilized by other services such as unicast.

Notice that our scheduler depends on both the queue state in terms of available slots for resource allocation at MAC layer, and the channel state at the physical layer. Hence, it offers cross-layer scheduler facilities.

\section{B. Traffic Modeling}

The services considered for scheduling analysis are given in Table I. For video streaming model, packet generation rate is $64 \mathrm{kbps}$ for file size of $50 \mathrm{kB}$. For background services, which is categorized as best effort traffic, generation rate is $32 \mathrm{kbps}$ and the file size is $100 \mathrm{kB}$. The packet error rate (PER) for both services are set to $10^{-2}$.

TABLE I

SERVICES AND TRAFFIC MODELS CONSIDERED IN SIMULATIONS

\begin{tabular}{ccccc}
\hline $\begin{array}{c}\text { Service } \\
\text { category }\end{array}$ & Service type & $\begin{array}{c}\text { Traffic } \\
\text { model }\end{array}$ & $\begin{array}{c}\text { File } \\
\text { transfer } \\
\text { delay }\end{array}$ & $\begin{array}{c}\text { Packet } \\
\text { error } \\
\text { rate } \\
\text { (PER) }\end{array}$ \\
\hline Streaming & $\begin{array}{c}\text { Video } \\
\text { streaming }\end{array}$ & $\begin{array}{c}\text { Generation } \\
\text { rate 64kbps, } \\
\text { file size } \\
50 \mathrm{kB}\end{array}$ & $0.01 \mathrm{~s}$ & $10^{-2}$ \\
\hline $\begin{array}{c}\text { File } \\
\text { delivery }\end{array}$ & $\begin{array}{c}\text { Software } \\
\text { distribution }\end{array}$ & $\begin{array}{c}\text { Generation } \\
\text { rate } 32 \mathrm{kbps}, \\
\text { file size } \\
100 \mathrm{kB}\end{array}$ & $1 \mathrm{~s}$ & $10^{-2}$ \\
\hline & & & \\
\hline
\end{tabular}

\section{Channel Model}

The reference link level simulation curve, relating PER vs $\mathrm{Eb} / \mathrm{No}$ is as given in [6]. The reference curve uses Turbo coding, where the physical layer adaptation is observed and the performance is reflected in PER employing a Rician channel with $\mathrm{K}$ factor of between 0 and $7 \mathrm{~dB}$. The system employs time-division multiple access (TDMA) and focuses on downlink transmission, although our results can be extended to the uplink transmission as well. Notably, the Rician channel is representative of rural or suburban environments. In transmitting file delivery services, the desired PER, which is $10^{-2}$, can be achieved by $E_{b} / \mathrm{N}_{0}$ values below the desired PER. The entire Eb/No values are partitioned into non-overlapping consecutive intervals such that the range is fixed every $0.25 \mathrm{~dB}$. 
The signal measured at the user is defined as $s(t)$ which is calculated as

$$
s(t)=s_{\text {random }}(t)+\mu
$$

where $\mu$ is the mean value and $s_{\text {random }}(t)$ is defined as

$$
s_{\text {random }}(t)=A x s_{\text {random }}(t-1)+\sqrt{1-A^{2}} x s_{\text {random_uncor }}(t)
$$

where $A$ is the auto-correlation factor and $s_{\text {random_uncor }}$ is defined as

$s_{\text {random_uncor }}=\sigma \times w g n(t)$

where $\sigma$ is sigma and $w g n(t)$ is white Gaussian noise random coefficient.

Referring to [11], single environment model uses values for $\mu$ and $\sigma$ calculated for urban areas with $\mathrm{K}$ factor of 7 and elevation angle $\alpha$ of $80^{\circ}$. The values are 1.7480 for $\mu$ and 0.8 for $\sigma$. The auto-correlation factor, $A$, is set to 0.8 for both single and multi-environment. Whereas for multi-environment, different values of $K$ are reflected on specified mean and standard deviation values, as will be discussed further in Section III(b).

Notably, it is assumed that the channel remains invariant per packet for each user. However the channel is allowed to vary from packet to packet. We also assume that perfect channel state information (CSI) is available at the receiver. This information is fed back to the satellite without latency and error.

\section{Performance Evaluations AND CONCLUSion}

\section{A. Single Environment}

Our goal here is to reduce the number of retransmission due to bad channel conditions. The initial result shown in Fig. 2 depicts the scheduler's performance for delay-tolerant traffic from file delivery service both with and without the employment of cross-layer design. The figure is meant for validation and verification of the simulator as to whether the simulator giving the correct result or not.

Here, failure rate is calculated in terms of fraction of multicast users in a multicast group requesting retransmission. The failure rate is directly related to packet loss rate (PLR) due to bad channel condition. The packet loss rate is plotted over the number of users in a multicast group. For cross layer scheduling the threshold fraction of users to resume transmission, $\varsigma_{T}$, is 0.9 and 0.8 . It is observed that by deploying channel state information on a multicast packet scheduler, the packet loss rate is reduced significantly. This is because in the scheduler with channel information, the chosen packet has relatively high percentage of 'good' channel condition. Thus packet loss due to bad channel condition is higher in scheduler without channel information compared to scheduler with channel information. The behavior of PLR is almost constant with multicast group size because the fraction of multicast users in a group having bad channels is the same ratio and directly related to the target packet error rate. Furthermore, we assume that all users experiences the same environment, that is Rician distribution with $\mathrm{K}$ factor of $7 \mathrm{~dB}$.

It is seen that high success rate is achieved by low retransmission requests by users which is an advantage for multicast service delivery as well as preserving the network resources. Since the scheduler consideration is for non-delay sensitive service, this does not affect the performance of delaysensitive traffic, such as streaming traffic.

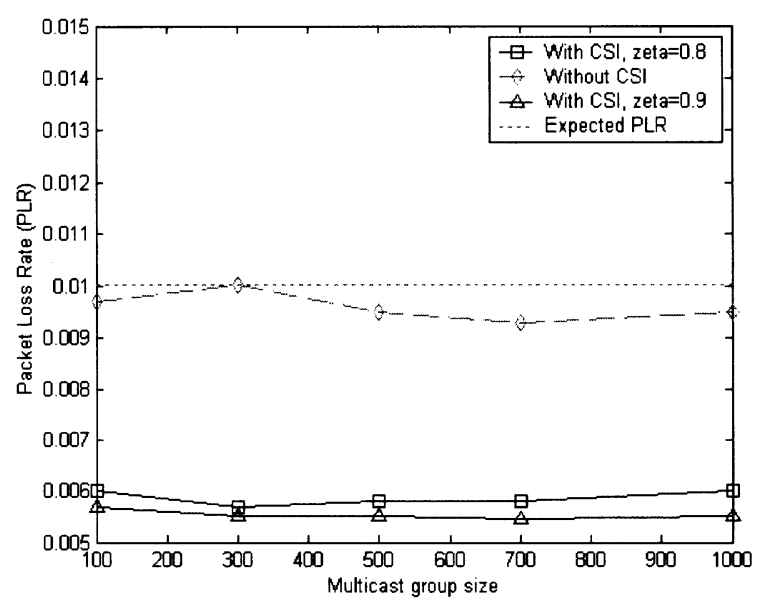

Fig. 2 Packet Loss Rate vs. multicast group size

To verify the expected packet loss rate (PLR), equation (13) in [9] is used:

$P L R=1-\left(1-P_{d}\right)\left(1-P_{0}\right)$

Where $P_{d}$ denotes packet dropping due to overflow or blocking probability and $P_{0}$ is the target PER which is set to $10^{-2}$ in this paper. Since we focus on the impact of shadowing in physical layer, we assume there is no packet to be dropped due to overflow, i.e., $P_{d}$ is 0 .

Now extending the investigations in the scope of cross-layer scheduling, the percentage threshold is varied to observe the performance of the scheduler. Again in Fig. 2, it is observed that lower value of $\varsigma_{\mathrm{T}}$ (zeta), experiences higher packet loss rate. Increasing the threshold is not harmful since the transmission is more guaranteed in this group than in groups with low percentage threshold. As we take the probability of at least one user request retransmission due to bad channel condition, it is observed in Fig. 3 that larger multicast group sizes suffer high probability of retransmission. The probability is defined as

$P\left\{n_{R t x}>=1\right\}=\frac{n_{b a d}}{N}$

where $n_{b a d}$ is the number of bad channel events and $N$ is the total number of events.

The increase of probability of retransmission with respect to multicast group size occurs because the fraction of users in bad channel conditions is linearly related to a multicast group size. From Fig. 3, it is shown that by increasing $\varsigma_{T}$ (zeta), the 
probability can be substantially reduced. Thus $\varsigma_{T}$ becomes a control parameter to monitor failure rate in certain multicast group size.

However, by increasing $\varsigma_{T}$ (zeta), the average packet delay is increased. This is depicted in Fig. 4. The higher the percentage, the longer a multicast group has to wait for packets to be transmitted to them. Fig. 4 considers average packet delay due to both delays waiting for slots as well as delay due to waiting for good channel condition,

$\tau=\tau_{C h}+\tau_{q}$

However, we assume the number of slots is infinite as we want to show the impact exclusively on delay due to waiting for achieving high fraction of users with good channel condition only.

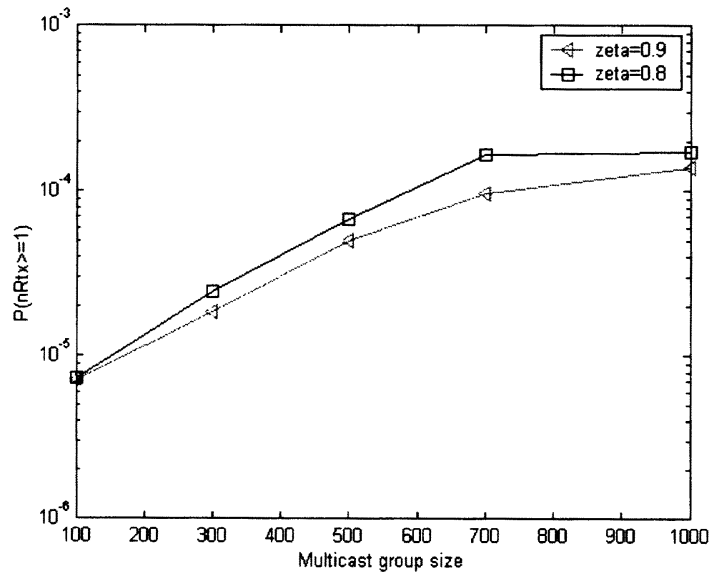

Fig. 3 Probability of at least one user in a multicast group reques retransmission (failure rate) vs. Eb/No threshold

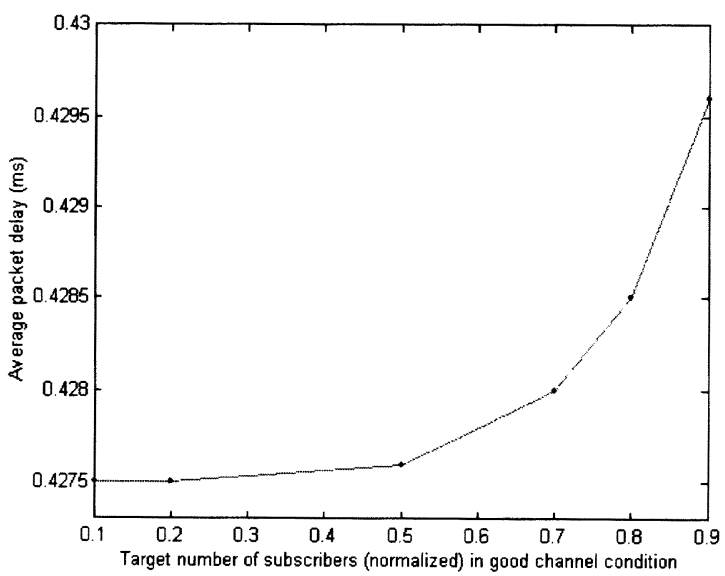

Fig. 4 Average packet delay vs. target number of users (normalized) in good channel cond ition, $\varsigma_{T}(z e t a)$

TABLE II

SIMULATION PARAMETERS FOR AVERAGE PACKET DELAY
Multicast group size

Maximum re-attempt 000

$50 * 20 \mathrm{~ms} / \mathrm{slot}$

\section{B. Multi-channel Environment}

Next, we model different channel conditions to represent different environments experienced by users in a multicast group. For each considered environment, by means of empirical models from [11], the optimum values for Rician factor, $K$, mean, $\mu$, and standard deviation, $\sigma$, as functions of elevation angle, $\alpha$, are

$$
\begin{aligned}
& K=K_{0}+K_{1} \alpha+K_{2} \alpha^{2}+K_{3} \alpha^{3} \\
& \mu=\mu_{0}+\mu_{1} \alpha+\mu_{2} \alpha^{2}+\mu_{3} \alpha^{3} \\
& \sigma=\sigma_{0}+\sigma_{1} \alpha+\sigma_{2} \alpha^{2}+\sigma_{3} \alpha^{3}
\end{aligned}
$$

The coefficients are provided in Table II for considered environmental conditions. The polynomial empirical formulas are valid in the range $20^{\circ}<\alpha<90^{\circ}$.

TABLE II

EMIPIRICAL COEFFICIENTS FOR DIFFERENT ENVIRONMENTS [11]

\begin{tabular}{|c|c|c|}
\hline Coefficient value & Suburban area & Urban area \\
\hline $\mathrm{K}_{0}$ & -13.600 & 1.750 \\
\hline $\mathrm{K}_{1}$ & $9.650^{*} 10^{-1}$ & $6.700^{*} 10^{-2}$ \\
\hline $\mathrm{K}_{2}$ & $-1.663^{*} 10^{-2}$ & 0.0 \\
\hline $\mathrm{K}_{3}$ & $1.187^{*} 10^{-4}$ & 0.0 \\
\hline$\mu_{0}$ & -1.998 & -52.12 \\
\hline$\mu_{1}$ & $-9.919^{*} 10^{-3}$ & 2.758 \\
\hline$\mu_{2}$ & $1.520^{*} 10^{-3}$ & $-4.777^{*} 10^{-2}$ \\
\hline$\mu_{3}$ & $-1.266^{*} 10^{-5}$ & $2.714^{*} 10^{-4}$ \\
\hline$\sigma_{0}$ & 8.000 & 7.800 \\
\hline$\sigma_{1}$ & $-3.741^{*} 10^{-1}$ & $-3.542^{*} 10^{-1}$ \\
\hline$\sigma_{2}$ & $6.125^{*} 10^{-3}$ & $6.500^{*} 10^{-3}$ \\
\hline$\sigma_{3}$ & $-3.333^{*} 10^{-5}$ & $-3.958^{*} 10^{-5}$ \\
\hline
\end{tabular}

In this scenario, the percentage of users in suburban and urban area is stated in Table III. The Rician $\mathrm{K}$ factor is measured in $\mathrm{dB}$ and defined as the ration of power in constant part and power in random part of Rician distribution. The mean, $\mu$, and standard deviation, $\sigma$, are parameters of Rician probability density function (pdf). The values of each parameters are presented in Table III, calculated from empirical models in equations (7)-(9).

TABLE III

SIMULATION PARAMETERS FOR MULTI-ENVIRONMENT SCENARIO

\begin{tabular}{|c|c|c|c|c|c|}
\hline Area & $\begin{array}{c}\text { Rician } \boldsymbol{K} \\
\text { factor }\end{array}$ & $\alpha(\rho)$ & $\mu$ & $\boldsymbol{\sigma}$ & $\begin{array}{c}\% \\
\text { Subs }\end{array}$ \\
\hline Suburban & $0 \mathrm{~dB}$ & 20 & -1.69 & 2.70 & 20 \\
\hline Urban & $3 \mathrm{~dB}$ & 20 & -13.90 & 3.06 & 40 \\
\hline Urban & $7 \mathrm{~dB}$ & 80 & 1.75 & 0.80 & 30 \\
\hline Suburban & $10 \mathrm{~dB}$ & 60 & 0.14 & 0.40 & 10 \\
\hline \hline
\end{tabular}

The operating point is a defined $\mathrm{Eb} / \mathrm{No}$ value for each user, which is given by

$\gamma_{i}(t)=\gamma_{r e f}-\Gamma_{i}(t)$

where $\gamma_{\text {ref }}$ is the reference $\mathrm{Eb} / \mathrm{No}$ from the AWGN channel reference model to achieve target PER of $10^{-2}$ and $\Gamma_{i}(t)$ is the calculated received signal, $s(t)$, for each user $i$ in the multicast group using $\mu$ and $\sigma$ values stated in Table III . 
In this scenario, the packet size is set to 125 bytes and multicast group size is 1000 users. The algorithm for multienvironment is the same as in single environment except that instead of comparing the achieved PER with the target PER, we now compare the achieved operating point, $\gamma_{i}(t)$, with Eb/No threshold, $\gamma_{T}$.

With the mixture of environments in the scenario, the probability of at least one user request retransmission is plotted vs. $\gamma_{T}$. The result is compared over fraction of users in relatively good channel, $\varsigma_{T}(z e t a)$. From Fig. 5 , as $\gamma_{T}$ increases, the probability of retransmission decreases due to the high threshold that each user has to meet in order to be considered for transmission. Furthermore, higher $\varsigma_{T}$ (zeta) experiences lower failure rate, reflecting a second threshold that the multicast group has to meet prior to obtaining a slot from multicast scheduler for downlink transmission.

As opposed to low probability of retransmission enjoyed in higher values of $\gamma_{T}$, high average packet delay is expected as the price to pay for waiting for a good fraction of users in the group, $\varsigma_{i}$, having good channels, as depicted in Fig. 6. This is because, the packet intended for multicast group which does not meet $\varsigma_{T}$ in the first attempt, is retracted (back-off) and repeated until it reaches $\varsigma_{i}$ higher than $\varsigma_{T}$. The repeat mechanism increases the waiting time of the multicast packet.

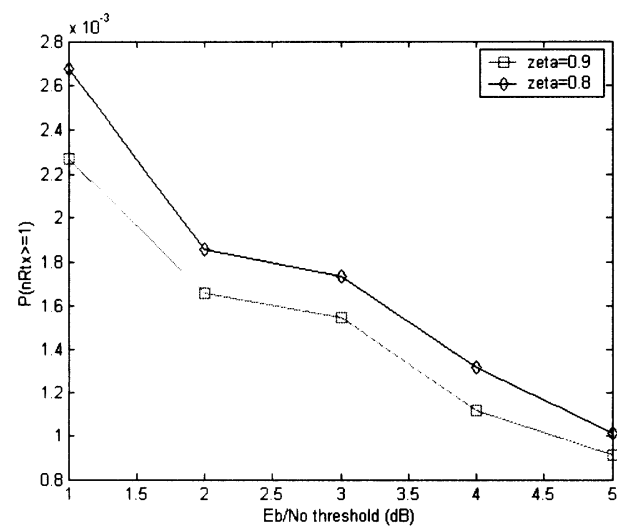

Fig. 5 Probability of at least one user in a multicast group request retransmission (failure rate) vs. Eb/No threshold, $\gamma_{T}$

\section{CONCLUSIONS}

This paper assumes single and multi-environment satellite channels for downlink transmission of multicast packets with cross layer design mechanism. Channel state information (CSI) of each user in the multicast group is considered and becomes the condition for the transmission of the multicast packets. The observations are sustained in best effort traffic though the scenario simulates real-time traffic as well. The result indicates that a positive performance improvement is gained by adopting a cross-layer-design approach in fading environments.

By obtaining the CSI before transmitting multicast flows, the approach reduces unnecessary transmission of background traffic and hence reduces unnecessary resource allocation and retransmission requests. However, to achieve a relatively good channel condition for a multicast group, higher average packet delay is expected. For further work, more accurate channel estimation to depict real-environments will be investigated for scheduling and transmission of multicast packets.

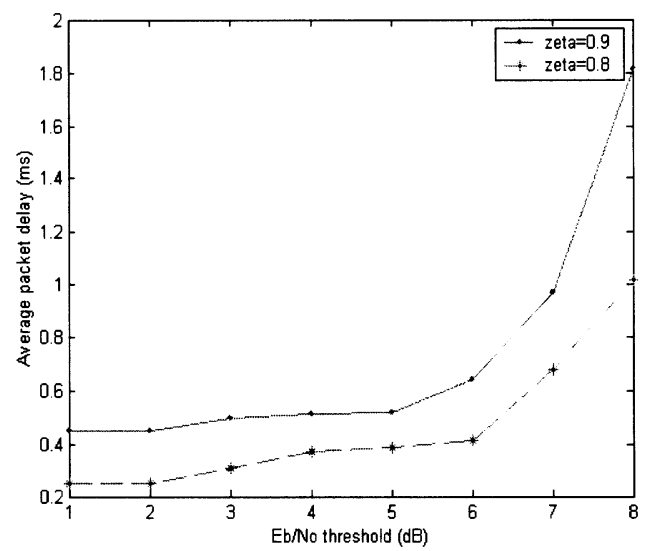

Fig. 6 Average packet delay vs. Eb/No threshold, $\gamma_{T}$

\section{REFERENCES}

[1] J. Zhang, "Bursty traffic meets fading: a cross-layer design perspective," Proceeding of the 40th Allerton Conference on Communications, Control, and Computing, Oct. 2002

[2] H.P.Lin, M.J.Tseng, 'Two-Level Multistate Markov for Satellite Propagation Channel', IEE Proceedings Microwave and Antenna Propagation, Vol. 151, No. 3, June 2004

[3] M. Karaliopoulos, P. Henrio, K. Narenthiran, E. Angelou, B.G.Evans, 'Packet Scheduling for the Delivery of Multicast and Broadcast Services over S-UMTS', International Journal of Satellite Communication Network, 2004

[4] T.Stockhammer, M.H.Hannuksela, T.Wiegand, 'H.264/AVC in Wireless Environments', IEEE Transactions on Circuits and Video Telephony, Vol. 13, No. 7, July 2003

[5] Q.Liu, S.Zhou, G.B.Giannakis, 'Cross-Layer Scheduling With Prescribed QoS Guarantees in Adaptive Wireless Networks', IEEE Journal in Selected Areas in Communications, Vol. 23, No. 5, May 2005

[6] S. Papaharalabos, P. Sweeney, and B. G. Evans, "A New Method of Improving SOVA Turbo Decoding for AWGN, Rayleigh and Rician Fading Channels", IEEE VTC 2004-Spring, Vol. 5, 17-19 May 2004, Milan, Italy, pp. 2862-2866

[7] F.Ananasso, F.Valataro, 'Mobile and Personal Satellite Communications', Springer-Verlag London Ltd., 1995, pp225-250

[8] M.A.Haleem, R.Chandramouli, 'Adaptive Downlink Scheduling and Rate Selection: A Cross-Layer Design', IEEE Journal in Selected Areas in Communications, Vol. 23, No. 6, May 2005

[9] Q.Liu, S.Zhou, G.B.Giannakis, 'Queuing With Adaptive Modulation and Coding Over Wireless Links: Cross-Layer Analysis and Design', IEEE Transactions on Wireless Communications, Vol. 4, No. 3, May 2005

[10] S.R.Saunders, 'Antennas and Propagation for Wireless Communication Systems', John Wiley\&Sons, 1999, pp180-188, pp215-220

[11] G.E.Corazza,A.Jahn, E.Lutz, F.Valataro, 'Channel Charanterization for Mobile Satellite Communications', Proceedings of the First European Workshopon Mobile/Personal Satcoms (EMPS94) 